\title{
Self-care ability and influencing factors in chronic heart failure patients.
}

\author{
Xiaoling Lei ${ }^{1, *}$, Minsui Cai ${ }^{2}$ \\ ${ }^{1}$ School of Nursing, Nantong University, \#19 Qixiu Road, Nantong 226001, PR China \\ ${ }^{2}$ Ultrasonic Department, Affiliated Hospital of Nantong University, \#20 Xisi Road, Nantong 226001, PR China
}

\begin{abstract}
Objectives: To describe the self-care abilities and associated factors in chronic heart failure (CHF) patients.

Methods: Participant characteristics, illness-related information, and self-care ability were investigated using self-report questionnaires and patient records for 91 CHF patients in Nantong University Affiliated Hospital, China.

Results: On average, patients had medium- to low-level self-care abilities. Comprehensive self-care ability was positively influenced by higher education level, employment status, and income level. Patients who received health education and had sound heart function had better self-care abilities. Regression analysis showed that the factors influencing self-care ability included average monthly income, education level, and heart function grading.

Conclusions: Self-care ability in CHF patients is, in general, in medium to low ranges, indicating that medical workers need to enhance self-care education for CHF patients to help them better understand their condition and help them gain the necessary skills for self-care. We conclude that, when conducting self-care education for patients, medical workers should tailor their approach based on the individual characteristics of the patient. Doing so would effectively promote patient knowledge, mobility, and treatment compliance.
\end{abstract}

Keywords: Cardiac heart failure, Self-care ability, Influencing factor.

Accepted on November 13, 2017

\section{Introduction}

Chronic heart failure (CHF) has become a prominent global public health issue, with a worldwide prevalence rate of around 1-2\%. Patients with CHF face severe threats to their health, a reduced capacity to work, a poorer quality of life, and place a sizable strain on medical resources, resulting in a heavy financial burden for affected households and society in general [1]. In recent years, with advancements in pathophysiology, new strategies have been advanced for the treatment of CHF, yet the admission rate of CHF patients keeps climbing [2]. Therefore, to improve the quality of life for CHF patients, treatment goals should lie not only in improving symptoms, but also in lowering the rates of readmission and fatalities. Previous studies have verified that self-care can improve $\mathrm{CHF}$ patient prognosis $[3,4]$, and that effective self-care could improve the health of patients [5], improve quality of life [6], and reduce the number of hospital admissions as well as fatality rates [7-9]. However, the self-care abilities of $\mathrm{CHF}$ patients have been reported to be poor to moderate [10]. Considering these findings, the current study aims to gain a better insight into the self-care abilities of CHF patients and the factors influencing self-care, with a view to raising nurses' awareness of issues impacting self-care in patients and helping nurses determine the degree of compensatory nursing required. Based on the results of a survey analysis of 91 cases of selfcare in CHF patients, a support-education method is proposed as a means of educating patients to improve their self-care abilities.

\section{Materials and Methods}

\section{Participants}

This research involved 91 CHF patients from the Vasculocardiology Department of Nantong University Affiliated Hospital in China, and was conducted between June 2015 and August 2016. A convenience sampling methodology was employed, and self-report questionnaires were administered to 93 patients, with 91 complete questionnaires being returned, resulting in a valid return rate of $97.85 \%$. Inclusion criteria were as follows: (1) inpatients with a confirmed diagnosis of CHF given by the doctor in charge or senior physicians; (2) New York Heart Association (NHYA) cardiac function grading II-IV class; (3) the basic disease leading to heart failure was a disease of the cardiovascular system; (4) being able to independently complete questionnaires (written or oral); and (5) being willing to take 
part in the survey. Exclusion criteria were: (1) patients in the terminal stage of CHF with cachexia; (2) complications involving severe diseases in other systems; (3) cognitive dysfunction; (4) not being willing to participate.

The study protocol was approved by the Ethics Committee of Nantong University Affiliated Hospital, and patients who participated in the questionnaire survey all signed informed consents. Participants were all informed about their right to withdraw from the study, and they were told that their information was to be kept confidential and anonymous, and to be used for no other purpose.

\section{Methods}

Two undergraduate nurses administered surveys as part of their course requirements. After obtaining informed consent from patients in stabilized conditions, self-report questionnaires were administered. If participants were not able to complete the questionnaires themselves, family members or medical workers could help on their behalf, but did not offer any guidance regarding how to answer questions. If participants had any queries as to how to complete the questionnaires, they were able to seek help from the undergraduate nurses. After completion, questionnaires were collected on the spot.

\section{Survey tools}

General participant characteristics were recorded, including basic information such as age, sex, education level, monthly income, and marital status. Information relating to illness duration and cardiac function grading was obtained through patient records. Cardiac function was graded according to the 1928 standards set out by the New York Heart Association.

This study utilized a modified CHF patient comprehensive self-care ability evaluation scale questionnaire (MCHFCSQ) [10]. The scale contains 20 items divided across 3 dimensions: patient knowledge of CHF (information subscale), self-care ability (mobility subscale), and compliance behaviour in $\mathrm{CHF}$ patients (treatment compliance subscale). All entries were scored as 0,3 , or 5 . The total scores for the MCHFCSQ are as follows: whole measure scores range between $0-100$, the information subscale scores range between 0-40, mobility subscale scores range between $0-30$, and treatment compliance subscale scores range between 0-30. Higher scores indicate stronger comprehensive self-care ability and better self-care. Comprehensive self-care ability is divided into high, medium, and low levels based on cut off scores of $>85$ points, $70-85$ points, and $<70$ points, respectively. In 2007 , Liu [11] reported the Cronbach's $\alpha$ coefficient of the scale and the three dimensions as being 0.846, 0.840, 0.695, and 0.654, respectively. Communality was reported as being $88.3 \%$, indicating high levels of reliability and validity. It is not necessary to evaluate Cronbach's $\alpha$ for validated questionnaires, because their reliability has already been confirmed [11]. While the targets of previous research and our study differed, similar sets of questions were presented to participants. Thus, we did not assess Cronbach's $\alpha$ in our study as the validity of our scale was guaranteed.

\section{Statistical methods}

Epidata 3.02 was used for electronically recording participants' questionnaire responses, and SPSS 21.0 for Windows was utilized for statistical analysis. Descriptive statistics, such as means, standard deviations, frequency, and percentages were obtained for sociodemographic data and patient records. Chisquare tests, t-tests, and ANOVAs (or rank sum multiple comparison tests) were conducted to compare comprehensive self-care abilities by sociodemographic and illness-related variables, such as age and sex, and to analyse differences across the three MCHFCSQ dimensions. Stepwise linear regression, or ordinal regression, was adopted to analyse the factors influencing comprehensive self-care abilities in $\mathrm{CHF}$ patients. The threshold for statistical significance was set at .05 for all analyses.

\section{Results}

\section{Participant characteristics}

The $91 \mathrm{CHF}$ patients involved in this study had an average age of $65.94 \pm 15.60$ years, and $56.7 \%$ of participants were male. Regarding level of education, $24.7 \%$ of patients had received nine-years of compulsory education or gone on to further education. The majority of patients $(86 / 91)$ reported that they had received health education related to CHF from medical workers. Specific sociodemographic and disease related information can be found in Table 1 .

Table 1. Sociodemographic and illness-related patient characteristics $(n=91)$.

\begin{tabular}{|c|c|c|}
\hline Variable & Mean \pm standard deviation & Frequency (\%) \\
\hline Age & $65.94 \pm 15.60$ & \\
\hline \multicolumn{3}{|l|}{ Gender } \\
\hline Male & & $51(56.7)$ \\
\hline Female & & $39(43.3)$ \\
\hline \multicolumn{3}{|l|}{ BMI } \\
\hline$<18.5$ & & $8(8.9)$ \\
\hline $18.5-23.9$ & & $50(55.6)$ \\
\hline $24-27.9$ & & $22(24.4)$ \\
\hline$>28$ & & $10(11.1)$ \\
\hline \multicolumn{3}{|l|}{ Marital status } \\
\hline Married & & $75(82.4)$ \\
\hline Others & & $16(17.6)$ \\
\hline \multicolumn{3}{|l|}{ Residence } \\
\hline City & & $61(64.0)$ \\
\hline Countryside & & $22(24.7)$ \\
\hline
\end{tabular}




\begin{tabular}{ll}
\hline Education level & \\
\hline$<9$ years & $67(75.3)$ \\
\hline$>9$ years & $22(24.7)$ \\
\hline Employment status & \\
\hline Employment & $57(64.0)$ \\
\hline Unemployment & $32(36.0)$ \\
\hline Household average monthly income & \\
\hline$<1000$ RMB & $19(20.9)$ \\
\hline $1000-3000$ RMB & $34(37.4)$ \\
\hline$>3000$ RMB & $38(41.8)$ \\
\hline Medical insurance, yes & $78(85.7)$ \\
\hline IIIness duration & \\
\hline Admission times & $13(14.9)$ \\
\hline One time & \\
\hline$>$ one time & $36(94.08$ \\
\hline Mastery over CHF information or not, yes & $31(34.8)$ \\
\hline Caregiver & $58(65.2)$ \\
\hline Single caregiver & $62(72.1)$ \\
\hline Several caregivers & \\
\hline No caregiver & \\
\hline Reception of health education or not; yes & \\
\hline Cardiac function grading & \\
\hline II grade & \\
\hline III grade & \\
\hline Type grade & \\
\hline
\end{tabular}

Table 2. CHF patient comprehensive self-care ability and scores on MCHFCSQ subscales.

\begin{tabular}{|c|c|c|c|}
\hline & $\begin{array}{l}\text { Mean } \pm \text { standard } \\
\text { deviation }\end{array}$ & Minimum & Maximum \\
\hline $\begin{array}{l}\text { Comprehensive self- } \\
\text { care ability }\end{array}$ & $63.76 \pm 16.32$ & 25.00 & 100.00 \\
\hline Information mastery & $19.24 \pm 9.12$ & 3.00 & 40.00 \\
\hline Mobility & $23.05 \pm 5.6$ & 3.00 & 30.00 \\
\hline $\begin{array}{l}\text { Treatment } \\
\text { compliance }\end{array}$ & $21.46 \pm 5.67$ & 8.00 & 30.00 \\
\hline
\end{tabular}

\section{Self-care abilities in CHF patients}

CHF patients obtained an average score of $19.24 \pm 9.12$ on the information subscale of the MCHFCSQ, $23.05 \pm 5.60$ on the mobility subscale, and $21.46 \pm 5.67$ on the treatment compliance subscale. The lowest MCHFCSQ total score was
25 , and the highest was 98 points, with an average of $63.76 \pm$ 16.32. In general, the self-care abilities of CHF patients were in the low-medium range (Table 2).

\section{The influence of sociodemographic and illness-related factors on comprehensive self-care ability in $\mathrm{CHF}$ patients}

To examine the influence of sociodemographic and illnessrelated factors on self-care ability in CHF patients, we conducted a series of regression analyses. When all sociodemographic and illness-related factors (such as blood pressure and heart rate) were included in the regression model as independent variables, it was found that patient education level, employment status, average monthly income, level of health education, and cardiac function grading had a significant influence on patients' comprehensive self-care abilities $(\mathrm{P}<0.05)$. We then divided our sample into groups based on illness-related factors, such as cardiac function grading, to create three groups. We found that education level, employment status, and average monthly household income were all related to self-care abilities of CHF patients $(\mathrm{P}<0.01)$. The results are shown in Table 3.

Table 3. CHF patient comprehensive self-care ability score comparison across different sociodemographic and illness-related factors $(n=91)$; *: $P<0.05$; **: $P<0.01$.

\begin{tabular}{|c|c|c|c|c|c|c|}
\hline \multirow[t]{2}{*}{ Variable } & \multirow{2}{*}{$\begin{array}{l}\text { Comprehensiv } \\
\text { e self-care } \\
\text { ability }\end{array}$} & \multirow[t]{2}{*}{$\mathrm{P}$} & \multicolumn{2}{|c|}{$\begin{array}{l}\text { Comprehensive } \\
\text { ability }\end{array}$} & self-care & \multirow[t]{2}{*}{$\mathrm{P}$} \\
\hline & & & Low & Medium & High & \\
\hline Age & & & $\begin{array}{l}66.68 \pm \\
15.02\end{array}$ & $\begin{array}{l}65.77 \\
16.55\end{array}$ & $\begin{array}{l}61.38 \\
\pm \\
17.75\end{array}$ & 0.671 \\
\hline Gender & & 0.092 & & & & 0.312 \\
\hline Male & $66.61 \pm 14.72$ & & $\begin{array}{l}29 \\
(51.8)\end{array}$ & $\begin{array}{l}18 \\
(69.2)\end{array}$ & $\begin{array}{l}4 \\
(50.0)\end{array}$ & \\
\hline Female & $60.85 \pm 17.23$ & & $\begin{array}{l}27 \\
(48.2)\end{array}$ & $8(30.8)$ & $\begin{array}{l}4 \\
(50.0)\end{array}$ & \\
\hline BMI & & 0.75 & & & & 0.098 \\
\hline$<18.5$ & $66.50 \pm 22.41$ & & $4(7.1)$ & $2(7.7)$ & $\begin{array}{l}2 \\
(25.0)\end{array}$ & \\
\hline $18.5-23.9$ & $62.58 \pm 17.35$ & & $\begin{array}{l}34 \\
(60.7)\end{array}$ & $11(42.3)$ & $\begin{array}{l}5 \\
(62.5)\end{array}$ & \\
\hline $24-27.9$ & $63.00 \pm 14.16$ & & $\begin{array}{l}11 \\
(19.6)\end{array}$ & $\begin{array}{l}10 \\
(38.5)\end{array}$ & $\begin{array}{l}1 \\
(12.5)\end{array}$ & \\
\hline$>28$ & $65.40 \pm 11.87$ & & $\begin{array}{l}7 \\
(12.5)\end{array}$ & $3(11.5)$ & $0(0)$ & \\
\hline Marital status & & 0.48 & & & & 0.914 \\
\hline Married & $64.32 \pm 16.46$ & & $\begin{array}{l}46 \\
(80.7)\end{array}$ & $\begin{array}{l}22 \\
(84.6)\end{array}$ & $\begin{array}{l}7 \\
(87.5)\end{array}$ & \\
\hline Others & $61.13 \pm 15.91$ & & $\begin{array}{l}11 \\
(19.3)\end{array}$ & $4(15.4)$ & $\begin{array}{l}1 \\
(12.5)\end{array}$ & \\
\hline Residence & & 0.061 & & & & 0.378 \\
\hline
\end{tabular}




\begin{tabular}{|c|c|c|c|c|c|}
\hline City & $66.00 \pm 15.90$ & & $\begin{array}{l}36 \\
(63.2)\end{array}$ & $\begin{array}{l}18 \\
(87.5)\end{array}$ & $\begin{array}{l}7 \\
(87.5)\end{array}$ \\
\hline Countryside & $59.20 \pm 16.48$ & & $\begin{array}{l}21 \\
(36.8)\end{array}$ & $8(30.8)$ & $\begin{array}{l}1 \\
(12.5)\end{array}$ \\
\hline Education level & & $0.000^{* *}$ & & & $0.000^{* *}$ \\
\hline$<9$ years & $60.28 \pm 14.15$ & & $\begin{array}{l}48 \\
(87.3)\end{array}$ & $\begin{array}{l}18 \\
(69.2)\end{array}$ & $\begin{array}{l}1 \\
(12.5)\end{array}$ \\
\hline$>9$ years & $75.73 \pm 16.42$ & & $\begin{array}{l}7 \\
(12.7)\end{array}$ & $8(30.8)$ & $\begin{array}{l}7 \\
(87.5)\end{array}$ \\
\hline
\end{tabular}

\begin{tabular}{llllll}
\hline \multicolumn{2}{l}{ Employment status } & $0.000^{* *}$ & & & $0.002^{* *}$ \\
\hline Employment & $68.96 \pm 14.60$ & 28 & 21 & 8 \\
& & $(50.9)$ & $(80.8)$ & $(100)$ & \\
& & & & \\
Unemployment & $54.56 \pm 15.81$ & & $5(19.2)$ & $0(0)$ \\
& $549.1)$ & & &
\end{tabular}

Household average monthly $0.000^{* *}$ $0.000^{* *}$ income

\begin{tabular}{|c|c|c|c|c|c|c|}
\hline$<1000 \mathrm{RMB}$ & $50.63 \pm 13.15$ & & $\begin{array}{l}19 \\
(33.3)\end{array}$ & $0(0)$ & $0(0)$ & \\
\hline $\begin{array}{l}1000-3000 \\
\text { RMB }\end{array}$ & $63.97 \pm 14.02$ & & $\begin{array}{l}22 \\
(38.6)\end{array}$ & $\begin{array}{l}10 \\
(38.5)\end{array}$ & $\begin{array}{l}2 \\
(25.0)\end{array}$ & \\
\hline >3000 RMB & $70.13 \pm 16.07$ & & $\begin{array}{l}16 \\
(28.1)\end{array}$ & $\begin{array}{l}16 \\
(61.5)\end{array}$ & $\begin{array}{l}6 \\
(75.0)\end{array}$ & \\
\hline \multicolumn{2}{|c|}{ Medical insurance } & 0.858 & & & & 0.896 \\
\hline Yes & $63.88 \pm 16.61$ & & $\begin{array}{l}48 \\
(84.2)\end{array}$ & $\begin{array}{l}23 \\
(88.5)\end{array}$ & $\begin{array}{l}7 \\
(87.5)\end{array}$ & \\
\hline No & $63.00 \pm 15.09$ & & $\begin{array}{l}9 \\
(15.8)\end{array}$ & $3(11.5)$ & $\begin{array}{l}1 \\
(12.5)\end{array}$ & \\
\hline \multicolumn{2}{|l|}{ Illness duration } & & $\begin{array}{l}7.77 \pm \\
6.96\end{array}$ & $\begin{array}{l}4.76 \quad \pm \\
3.61\end{array}$ & $\begin{array}{l}8.73 \pm \\
13.35\end{array}$ & \\
\hline \multicolumn{2}{|c|}{ Number of hospital admissions } & 0.307 & & & & 1 \\
\hline One time & $66.16 \pm 14.33$ & & $\begin{array}{l}19 \\
(33.9)\end{array}$ & $9(36.0)$ & $\begin{array}{l}3 \\
(37.5)\end{array}$ & \\
\hline >one time & $62.40 \pm 17.47$ & & $\begin{array}{l}37 \\
(66.1)\end{array}$ & $\begin{array}{l}16 \\
(64.0)\end{array}$ & $\begin{array}{l}5 \\
(62.5)\end{array}$ & \\
\hline \multicolumn{2}{|c|}{ Mastery over CHF information } & 0.71 & & & & 0.873 \\
\hline Yes & $63.52 \pm 16.90$ & & $\begin{array}{l}39 \\
(68.4)\end{array}$ & $\begin{array}{l}18 \\
(69.2)\end{array}$ & $\begin{array}{l}5 \\
(62.5)\end{array}$ & \\
\hline No & $65.00 \pm 15.54$ & & $\begin{array}{l}18 \\
(31.6)\end{array}$ & $8(30.8)$ & $\begin{array}{l}3 \\
(37.5)\end{array}$ & \\
\hline Caregiver & & 0.353 & & & & 1 \\
\hline $\begin{array}{l}\text { Single } \\
\text { caregiver }\end{array}$ & $63.15 \pm 16.22$ & & $\begin{array}{l}33 \\
(57.9)\end{array}$ & $\begin{array}{l}16 \\
(61.5)\end{array}$ & $\begin{array}{l}5 \\
(62.5)\end{array}$ & \\
\hline $\begin{array}{l}\text { Several } \\
\text { caregivers }\end{array}$ & $65.22 \pm 16.51$ & & $\begin{array}{l}23 \\
(40.4)\end{array}$ & $\begin{array}{l}10 \\
(38.5)\end{array}$ & $\begin{array}{l}3 \\
(37.5)\end{array}$ & \\
\hline No caregiver & 44 & & $1(1.8)$ & $0(0)$ & $0(0)$ & \\
\hline \multicolumn{2}{|c|}{ Reception of health education } & $0.034^{*}$ & & & & 1 \\
\hline Yes & $64.63 \pm 15.72$ & & $\begin{array}{l}53 \\
(93.0)\end{array}$ & $\begin{array}{l}25 \\
(96.2)\end{array}$ & $\begin{array}{l}8 \\
(100)\end{array}$ & \\
\hline No & $48.80 \pm 21.16$ & & $4(7.0)$ & $1(3.8)$ & $0(0)$ & \\
\hline Cardiac funct & ding & $0.010^{*}$ & & & & 0.491 \\
\hline
\end{tabular}

\begin{tabular}{lllll}
\hline II grade & $66.68 \pm 15.44$ & $\begin{array}{l}17 \\
(31.5)\end{array}$ & $\begin{array}{l}10 \\
(40.0)\end{array}$ & $\begin{array}{l}4 \\
(50.0)\end{array}$ \\
\hline III grade & $65.84 \pm 15.96$ & $\begin{array}{l}26 \\
(48.1)\end{array}$ & $\begin{array}{l}13 \\
(52.0)\end{array}$ & $\begin{array}{l}4 \\
(50.0)\end{array}$ \\
\hline IV grade & $51.31 \pm 14.80$ & $\begin{array}{l}11 \\
(20.4)\end{array}$ & $2(8.0)$ & $0(0)$ \\
& & & \\
\hline Cardiovascular disease type & $1.74 \pm 1.81$ & $\pm 1.50 \pm 0.57$ \\
& & 0.77 & 0.63 & 0.53
\end{tabular}

\section{The influence of sociodemographic and illness-related} factors on the dimensions of self-care ability

CHF patient education level, employment status, household average monthly income, and level of health education, were all correlated with the information subscale of the MCHFCSQ $(\mathrm{P}<0.05)$. Sex, employment status, mastery over CHF information, and cardiac function grading were also correlated with CHF patient mobility $(\mathrm{P}<0.05)$. Treatment compliance was higher in CHF patients with higher levels of education, employment, high household average monthly income, and sound cardiac function, than in those with lower levels of education, unemployment, low household average monthly income, and poor cardiac function $(\mathrm{P}<0.05)$. Other sociodemographic and illness-related factors were not correlated with the information, mobility, and treatment compliance MCHFCSQ subscales in CHF patients $(\mathrm{P}>0.05)$, as shown in Table 4.

Table 4. Comparisons of different sociodemographic and illnessrelated factors in information mastery, mobility, and treatment compliance score, *: $P<0.05$; **: $P<0.01$.

\begin{tabular}{|c|c|c|c|c|c|c|}
\hline Variable & $\begin{array}{l}\text { Informatio } \\
\mathrm{n}\end{array}$ & $\mathrm{P}$ & Mobility & $\mathrm{P}$ & $\begin{array}{l}\text { Treatment } \\
\text { complianc } \\
\mathrm{e}\end{array}$ & $P$ \\
\hline Gender & & 0.614 & & $0.022^{*}$ & & 0.104 \\
\hline Male & $\begin{array}{l}19.84 \\
8.94\end{array}$ & \pm & $\begin{array}{l}24.37 \pm \\
4.54\end{array}$ & & $\begin{array}{l}22.39 \\
5.61\end{array}$ & \pm \\
\hline Female & $\begin{array}{l}18.87 \\
9.14\end{array}$ & \pm & $\begin{array}{l}21.54 \pm \\
6.39\end{array}$ & & $\begin{array}{l}20.44 \\
5.57\end{array}$ & \pm \\
\hline BMI & & 0.948 & & 0.688 & & 0.312 \\
\hline$<18.5$ & $\begin{array}{l}20.88 \\
10.95\end{array}$ & \pm & $\begin{array}{l}22.00 \pm \\
8.80\end{array}$ & & $\begin{array}{l}23.63 \\
5.34\end{array}$ & \pm \\
\hline $18.5-23.9$ & $\begin{array}{l}19.36 \\
9.79\end{array}$ & \pm & $\begin{array}{l}22.76 \pm \\
5.51\end{array}$ & & $\begin{array}{l}20.46 \\
5.69\end{array}$ & $t$ \\
\hline $24-27.9$ & $\begin{array}{l}19.27 \\
7.36\end{array}$ & \pm & $\begin{array}{l}23.23 \pm \\
5.39\end{array}$ & & $\begin{array}{l}22.50 \\
5.70\end{array}$ & \pm \\
\hline$>28$ & $\begin{array}{l}18.00 \\
8.84\end{array}$ & \pm & $\begin{array}{l}25.30 \pm \\
3.50\end{array}$ & & $\begin{array}{l}22.10 \\
5.78\end{array}$ & $t$ \\
\hline Marital status & & 0.807 & & 0.307 & & 0.155 \\
\hline Married & $\begin{array}{l}19.13 \\
9.26\end{array}$ & \pm & $\begin{array}{l}23.33 \pm \\
5.62\end{array}$ & & $\begin{array}{l}21.85 \\
5.62\end{array}$ & $t$ \\
\hline Others & $\begin{array}{l}19.75 \\
8.63\end{array}$ & \pm & $\begin{array}{l}21.75 \pm \\
5.47\end{array}$ & & $\begin{array}{l}19.63 \\
5.73\end{array}$ & $t$ \\
\hline Residence & & 0.148 & & 0.240 & & 0.060 \\
\hline
\end{tabular}




\begin{tabular}{|c|c|c|c|c|c|c|}
\hline City & $\begin{array}{l}20.21 \\
9.57\end{array}$ & \pm & $\begin{array}{l}23.54 \pm \\
4.92\end{array}$ & & $\begin{array}{l}22.25 \\
5.60\end{array}$ & \pm \\
\hline Countryside & $\begin{array}{l}17.27 \\
7.87\end{array}$ & \pm & $\begin{array}{l}22.07 \pm \\
6.77\end{array}$ & & $\begin{array}{l}19.87 \\
5.58\end{array}$ & \pm \\
\hline Education level & & $0.000^{* *}$ & & 0.068 & & $0.007^{* *}$ \\
\hline$<9$ years & $\begin{array}{l}17.01 \\
7.57\end{array}$ & \pm & $\begin{array}{l}22.54 \pm \\
5.75\end{array}$ & & $\begin{array}{l}20.73 \\
5.35\end{array}$ & \pm \\
\hline$>9$ years & $\begin{array}{l}26.32 \\
9.81\end{array}$ & \pm & $\begin{array}{l}25.05 \pm \\
4.74\end{array}$ & & $\begin{array}{l}24.36 \\
5.45\end{array}$ & \pm \\
\hline
\end{tabular}

\begin{tabular}{llllll}
\hline II grade & $\begin{array}{l}19.35 \\
9.67\end{array}$ & \pm & $\begin{array}{l}24.35 \pm \\
4.72\end{array}$ & $\begin{array}{l}22.97 \\
4.95\end{array}$ & \pm \\
\hline III grade & $\begin{array}{l}20.16 \\
8.68\end{array}$ & \pm & $\begin{array}{l}23.70 \pm \\
5.33\end{array}$ & $\begin{array}{l}21.98 \\
5.87\end{array}$ & \pm \\
& 15.77 & \pm & $\begin{array}{l}18.15 \pm \\
\text { IV grade }\end{array}$ & $\begin{array}{l}5.81 \\
17.38\end{array}$ & \pm \\
& 9.65 & & & 4.96 & \\
\hline
\end{tabular}

\section{Regression analysis of self-care abilities and influencing factors on MCHFCSQ dimensions}

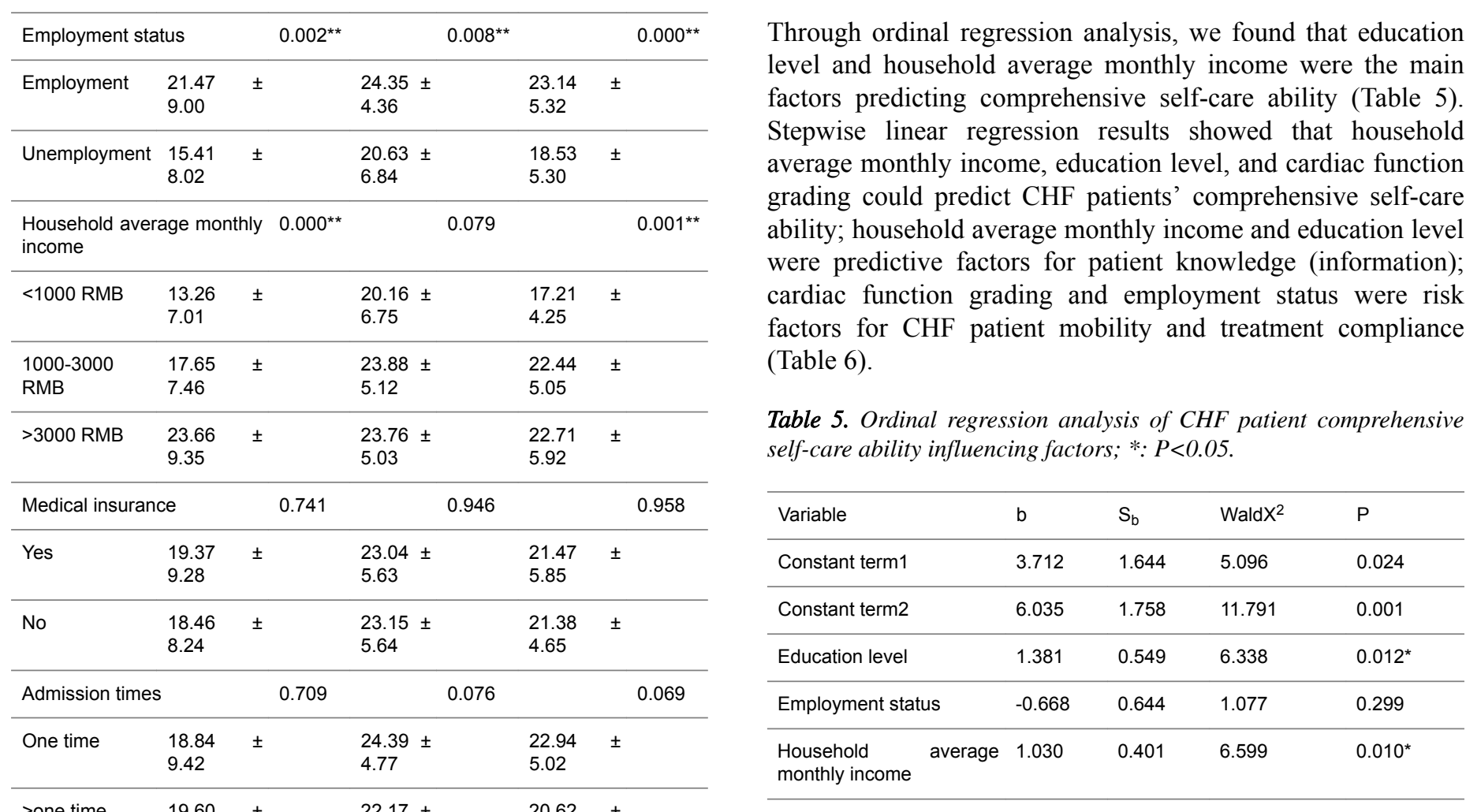

\section{Discussion}

\begin{tabular}{|c|c|c|c|c|c|c|}
\hline $\begin{array}{l}\text { Mastery ove } \\
\text { information or no }\end{array}$ & $\mathrm{CHF}$ & 0.457 & & $0.024^{*}$ & & 0.632 \\
\hline Yes & $\begin{array}{l}19.89 \\
8.50\end{array}$ & & $\begin{array}{l}22.40 \pm \\
6.02\end{array}$ & & $\begin{array}{l}21.23 \\
5.67\end{array}$ & \pm \\
\hline No & $\begin{array}{l}18.25 \\
10.56\end{array}$ & & $\begin{array}{l}24.83 \pm \\
3.56\end{array}$ & & $\begin{array}{l}21.92 \\
6.22\end{array}$ & \pm \\
\hline Caregiver & & 0.518 & & 0.641 & & 0.304 \\
\hline $\begin{array}{l}\text { Single } \\
\text { caregiver }\end{array}$ & $\begin{array}{l}18.74 \\
9.50\end{array}$ & & $\begin{array}{l}23.11 \pm \\
5.23\end{array}$ & & $\begin{array}{l}21.30 \\
5.66\end{array}$ & \pm \\
\hline $\begin{array}{l}\text { Several } \\
\text { caregivers }\end{array}$ & $\begin{array}{l}20.19 \\
8.59\end{array}$ & & $\begin{array}{l}23.08 \pm \\
6.23\end{array}$ & & $\begin{array}{l}21.94 \\
5.65\end{array}$ & \pm \\
\hline No caregiver & 12.00 & & 19.00 & & 13.00 & \\
\hline $\begin{array}{l}\text { Reception of } \\
\text { education or not }\end{array}$ & health & $0.032^{*}$ & & 0.344 & & 0.556 \\
\hline Yes & $\begin{array}{l}19.73 \\
9.02\end{array}$ & & $\begin{array}{l}23.35 \pm \\
5.08\end{array}$ & & $\begin{array}{l}21.55 \\
5.58\end{array}$ & \pm \\
\hline No & $\begin{array}{l}10.80 \\
6.61\end{array}$ & & $\begin{array}{l}18.00 \pm \\
11.11\end{array}$ & & $\begin{array}{l}20.00 \\
7.71\end{array}$ & \pm \\
\hline Cardiac function & grading & 0.370 & & $0.004^{* *}$ & & $0.012^{*}$ \\
\hline
\end{tabular}

The results of this study show that CHF patients with high selfcare abilities only account for $8.8 \%$ (8/91) of the total sample, with $62.6 \%(57 / 91)$ at the low level of self-care ability. The average score for patient comprehensive self-care ability was $63.76 \pm 16.32$, in the medium to low ability range, which is consistent with previous research $[12,13]$. We also found that education level, employment status, household average monthly income, level of health education, and cardiac function grading were related to comprehensive self-care ability in CHF patients; sex and knowledge of the illness also influenced mobility; and education level, household average monthly income, employment status, and cardiac function grading serve as independent predictive factors for comprehensive self-care ability, amount of knowledge about the illness/CHF, mobility, and treatment compliance. This indicates that those with lower levels of education, lower incomes, and who are unemployed should have more attention paid to them because they are more likely to not be as 
proficient at self-care. As such, targeted intervention measures are required to improve their self-care abilities.

Table 6. Stepwise linear regression analysis of $\mathrm{CHF}$ patient comprehensive self-care ability and influencing factors upon dimensions, *: $P<0.05$; **: $P<0.01$.

\begin{tabular}{|c|c|c|c|c|c|c|}
\hline & \multirow[t]{2}{*}{ Variable } & \multicolumn{2}{|c|}{$\begin{array}{l}\text { unstandardized } \\
\text { coefficient }\end{array}$} & \multirow{2}{*}{$\begin{array}{l}\text { standardiz } \\
\text { ed } \\
\text { coefficient }\end{array}$} & \multirow[t]{2}{*}{$\mathrm{t}$} & \multirow[t]{2}{*}{$P$} \\
\hline & & B & $\begin{array}{l}\text { Standard } \\
\text { deviation }\end{array}$ & & & \\
\hline \multirow{4}{*}{$\begin{array}{l}\text { Compreh } \\
\text { ensive } \\
\text { self-care } \\
\text { ability }\end{array}$} & Constant & $\begin{array}{l}44.13 \\
6\end{array}$ & 6.799 & & 6.492 & 0.000 \\
\hline & $\begin{array}{l}\text { Household } \\
\text { average } \\
\text { monthly } \\
\text { income }\end{array}$ & 7.067 & 2.041 & 0.343 & 3.463 & $0.001^{* *}$ \\
\hline & Education level & $\begin{array}{l}10.38 \\
9\end{array}$ & 3.675 & 0.280 & 2.827 & $0.006^{\star *}$ \\
\hline & $\begin{array}{l}\text { Cardiac } \\
\text { function } \\
\text { grading }\end{array}$ & -4.811 & 2.198 & -0.203 & -2.189 & $0.031^{*}$ \\
\hline \multirow{3}{*}{$\begin{array}{l}\text { Informati } \\
\text { on } \\
\text { mastery }\end{array}$} & Constant & 2.305 & 2.976 & & 0.774 & 0.441 \\
\hline & $\begin{array}{l}\text { Household } \\
\text { average } \\
\text { monthly } \\
\text { income }\end{array}$ & 3.946 & 1.124 & 0.340 & 3.510 & $0.001^{* *}$ \\
\hline & Education level & 6.715 & 2.013 & 0.323 & 3.335 & $0.001^{* *}$ \\
\hline \multirow[t]{3}{*}{ Mobility } & Constant & $\begin{array}{l}31.41 \\
6\end{array}$ & 2.092 & & 15.015 & 0.000 \\
\hline & $\begin{array}{l}\text { Cardiac } \\
\text { function } \\
\text { grading }\end{array}$ & -2.526 & 0.803 & -0.325 & -3.145 & $0.002^{* *}$ \\
\hline & $\begin{array}{l}\text { Employment } \\
\text { status }\end{array}$ & -2.640 & 1.169 & -0.233 & -2.259 & $0.027^{*}$ \\
\hline \multirow{3}{*}{$\begin{array}{l}\text { Treatme } \\
\mathrm{nt} \\
\text { complian } \\
\text { ce }\end{array}$} & Constant & $\begin{array}{l}30.94 \\
7\end{array}$ & 2.139 & & 14.466 & 0.000 \\
\hline & $\begin{array}{l}\text { Employment } \\
\text { status }\end{array}$ & -4.267 & 1.157 & -0.367 & -3.686 & $0.000^{* *}$ \\
\hline & $\begin{array}{l}\text { Cardiac } \\
\text { function } \\
\text { grading }\end{array}$ & -1.880 & 0.817 & -0.229 & -2.301 & $0.024^{*}$ \\
\hline
\end{tabular}

Domestic and foreign research shows that individual factors like sex, employment status, education level, and financial status had an influence on self-care ability [14]. Wang et al. [15] found that education level was positively correlated with patients' abilities to self-manage medication, treatments, and administration of medication, and that higher education levels could facilitate compliance with adaptive healthy behaviours. Hailan et al. [16] discovered that males tended to have a greater need for medical help at the onset of CHF than females, that patients in poorer health engaged in fewer activities, and that patients with low incomes had lower levels of self-care ability. O'Connell et al. [17] found that, through multi-centre studies of CHF patient self-care behaviours, patients with poor financial status had lower levels of self-care abilities. Siabani et al. [18] surveyed $225 \mathrm{CHF}$ patients using a cross-sectional method, and found that patient self-care ability was positively correlated with education level. We also found that education level, employment status, and average household monthly income influenced self-care ability, and that male patients with higher education levels, employment, and high average household monthly incomes, had better self-care abilities. This indicates that in educating patients about self-care, education should be tailored in accordance with individual participant characteristics

Previous studies showed that cardiac function grading, knowledge about the illness, and amount of health education could influence self-care ability. Cheng et al. [19] reported that patients with higher cardiac function gradings had worse cardiac function and were less able to live independently, which affected self-care ability to a degree. Rockwell et al. [20] reported that patients with higher cardiac function gradings experienced more symptoms, and that with worsening of cardiac function, patients understanding of CHF would grow deeper. Lv [21] also found that patient knowledge about CHF was related to cardiac function grading. Lv also reported that level of health education influenced patients' comprehensive self-care ability, and that patients who had received health education had better knowledge about their CHF, self-care abilities, and comprehensive self-care abilities than those who did not. Our research also found that self-care abilities were higher in patients who had received health education, who had low cardiac function gradings, and poor understanding of $\mathrm{CHF}$. This indicates that enhancing CHF patient health education could improve patient self-care ability and ultimately improve their quality of life.

Through this survey, we found that self-care abilities in $\mathrm{CHF}$ patients were generally poor, still in the medium to low range, and influenced by several factors. Future studies are required to further understand and verify the factors influencing self-care ability in CHF patients, with a view to ultimately informing the development of targeted intervention measures aimed at improving self-care abilities, and ultimately, quality of life for these patients.

\section{Statement of Ethical Approval}

The study protocol was approved in writing by the Ethics Committee of Nantong University Affiliated Hospital, and patients who participated in the questionnaire survey all signed informed consent forms. All participants were informed of their right to withdraw from the study, and were told that their information would be kept confidential and anonymous, and used for no other purpose.

\section{Declaration of Conflicting Interests}

The authors declare that there is no conflict of interest.

\section{Acknowledgements}

We thank Adam \& Stone Bio-Medicals Ltd Co. (Soochow, China) for language polishing. 


\section{References}

1. Rabelo ER, Aliti GB, Goldraich L, Domingues FB, Clausell N, Rohde LE. Non-pharmacological management of patients hospitalized with heart failure at a teaching hospital. Arq Bras Cardiol 2006; 87: 352-358.

2. Jessup M, Brozena S. Medical progress: Heart failure. N Engl J Med 2003; 348: 2007-2018.

3. Lee CS, Moser DK, Lennie TA, Riegel B. Event-free survival in adults with heart failure who engage in self-care management. Heart \& Lung 2011; 40: 12-20.

4. Riegel B, Lee CS, Dickson VV. Self-care in patients with chronic heart failure. Nat Rev Cardiol 2011; 8: 644-654.

5. Lee CS, Suwanno J, Riegel B. The relationship between self-care and health status domains in Thai patients with heart failure. Eur J Cardiovasc Nurs 2009; 8: 259-266.

6. Ditewig JB, Blok $\mathrm{H}$, Havers $J$, van Veenendaal $H$. Effectiveness of self-management interventions on mortality, hospital readmissions, chronic heart failure hospitalization rate and quality of life in patients with chronic heart failure: A systematic review. Patient Educ Couns 2010; 78: 297-315.

7. Steuten LM, Lemmens KM, Nieboer AP, Vrijhoef HJ. Identifying potentially cost effective chronic care programs for people with COPD. Int J Chron Obstruct Pulmon Dis 2009; 4: 87-100.

8. Sokol MC, McGuigan KA, Verbrugge RR, Epstein RS. Impact of medication adherence on hospitalization risk and healthcare cost. Med Care 2005; 43: 521-530.

9. Lorig KR, Sobel DS, Stewart AL, Brown BW, Bandura A, Ritter P, Gonzalez VM, Laurent DD, Holman HR. Evidence suggesting that a chronic disease self-management program can improve health status while reducing hospitalization A randomized trial. Med Care 1999; 37: 5-14.

10. Carlson B, Riegel B, Moser DK. Self-care abilities of patients with heart failure. Heart \& Lung 2001; 30: 351-9.

11. Liu X. Depression and Related Factors in Patients with Chronic Heart Fafture. Sun Yat-Sen University 2007.

12. Wang WN, Ding FF, Xing W, Si-Yi LI. Study on self-care behaviors and coping styles among chronic heart failure patients. Nurs Pract Res 2012.
13. Wang B, Wang J, Hua L, Wang Y, Zhu D, Liu S, Liu J. Relationship of Self-efficacy and Self-care Ability in Chronic Heart Failure Patients. Nursing Journal of Chinese People's Liberation Army 2015; 32: 25-28.

14. Siabani S, Leeder SR, Davidson PM. Barriers and facilitators to self-care in chronic heart failure: a metasynthesis of qualitative studies. Springerplus 2013; 2: 320.

15. Wang LP, Wang LZ. Effects of Community Nursing Intervention on Elderly Patients with Chronic Heart Failure. J Nurs 2009; 16: 1-3.

16. Hai-lan Q, Jun-qiao W, Chen S, Xiao-ling L. Self-care Behavior at Home of Patients with Chronic Heart Failure and Its Influencing Factors. J Nurs 2014; 21: 29-31.

17. Macabasco-O'Connell A, Crawford MH, Stotts N, Stewart A, Froelicher ES. Self-care behaviors in indigent patients with heart failure. J Cardiovasc Nurs 2008; 23: 223-30.

18. Siabani S, Driscoll T, Davidson PM, Najafi F, Jenkins MC, Leeder SR. Self-care and Its Predictors in Patients With Chronic Heart Failure in Western Iran. J Cardiovasc Nurs 2016; 31: 22-30.

19. Cheng X, Chen X, Chen J, Sujuan LU, Zhu Z. Effect of community nursing intervention on self-care of elderly patients with chronic heart failure. China Medical Herald 2014.

20. Rockwell JM, Riegel B. Predictors of self-care in persons with heart failure. Heart \& Lung 2001; 30: 18-25.

21. Lv F. Factors Influencing Self-care Agency of lnpatients with Chronic Heart FailureA Cross-Sectional Study. Shandong University 2011.

\section{*Correspondence to}

Xiaoling Lei

School of Nursing

Nantong University

PR China 